\title{
Solution of the Hard Problem of Consciousness
}

PHILOSOPHY, IDEAS

\section{S. Reinis}

Department of Psychology, University of Waterloo

Correspondence to: Stanislav Reinis, Ph.D.

Department of Psychology

University of Waterloo

Waterloo, Ontario, Canada

Key words:

mind, consciousness, quantum theory, Pauli principle

Activitas Nervosa Superior 2008;50:3, 48-57

Abstract

According to our hypothesis presented here, the human mind is a process depending on interactions of masses of mainly cortical neurons connected by biochemical, electrical and quantum factors into a massive system. The consciousness is dependent on the additional communication between particles, in particular between electrons. The Pauli repulsive exchange mechanism and other communication mechanisms may be able to transform physical events into a subjective information. 
Metzinger (1985) asked:

"How can consciousness arise in a physical universe? Is it at all imaginable that something like conscious experience could emerge from a purely physical basis? Is it conceivable that subjective sensations and the emergence of an inner perspective are part of the natural order or are we now confronted with an ultimate mystery, a grey area on the scientific map of the world that may in principle have to remain grey?

Today, the problem of consciousness - perhaps together with the question of the origin of the universe - marks the very limit of human striving for understanding. It appears to many to be the last great puzzle and the greatest theoretical challenge of our time. A solution of this puzzle through empirical research would bring about a scientific revolution of the first order."

David Chalmers, in his book "The Conscious Mind" (1997) has proposed that consciousness, like energy and mass, is a fundamental property of the universe and exists to varying degrees in all things, including the human brain. This is possible because the brain, as any other object in the universe, is formed by matter with all its general attributes. It is therefore important to search for elementary mechanisms which may be common both to the brain and the rest of the world.

There are several hypotheses explaining brain function and consciousness by quantum mechanisms. Since the very beginning of quantum mechanics, the founders of the theory claimed that the human mind depends on quantum events (e.g., Heisenberg, 1971). Several recent authors, such as Penrose, Stapp and Marshall, produced interesting theories of the mind and consciousness based on quantum mechanisms. These theories are, however, not generally accepted.

In this paper, we describe several elementary mechanisms of brain function, some of which cannot be explained by classical neuronal theory, and subsequently, we derive our proposal of the quantum hypothesis of the mind and consciousness from them. Some ideas described in this paper were published in 2005 (Reinis et al., 2005).

\section{Definition of consciousness as used in this paper}

There are many differences of opinion about the definition of consciousness based mainly on each authors'philosophy. Fortunately, in our considerations the definition of consciousness needs not be final - it seems that ultimately, after the solution of several main problems, there will be just one definition of consciousness accepted. We also believe that the division of opinions about consciousness into materialism and dualism is essentially prescientific and does not have to be discussed here. A complex of interactions affecting all components of the brain we consider a human mind whereas consciousness itself is a part of the mind expressing a subjective sense of self.

\section{Generation of submicroscopic particles in the brain}

Several submicroscopic particles participate in neuronal activities. Those are electrons, ions such as sodium, potassium, calcium, chloride and bicarbonate, larger molecules such as neurotransmitters, and even larger molecules such as brain peptides. In the explanation of the mechanism of mind and consciousness, electrons are probably more important than any other particle.

During the resting state of the neuronal membrane, this membrane is polarized due to the presence of several anions and cations, in particular proteins, sodium and potassium. Beside that, many free electrons bound to the inner and outer surfaces of the neuronal membrane are kept in shallow "potential wells". The potential wells are associated with electrically charged portions of protein molecules attached to the outer and inner surfaces of the cell membrane and with the polarized heads of lipids. Those electrons, named exoelectrons, contained in a potential well and possessing energy less than that required for escape from the well will remain in that well. A particle bound in a potential well may, under some circumstances, be pushed out from that well and be eventually found at a point distant from the well. In other words, when energy is added to such a particle, the particle escapes the well.

That happens during depolarization of the neuronal membrane. The differences between the electrical potentials on both sides of the neuronal membrane and between neighboring segments of the membrane produce an electric current, known as a circulating current. Circulating currents are formed by electrons released from the potential wells by a potential difference between segments of the axonal membrane. The circulating currents, e.g., those between two nodes of Ranvier, contain a large number of free electrons (Huxley, 1964).

In the formulation of the presented working hypothesis, all electric phenomena in the neuron must be considered, not just those that take place on the surface of the brain cells, in the cell membrane, but also those that act on the inside, in microtubules and mitochondria, as well as those involved in the conformation of protein molecules. All of them together represent a powerful source of subatomic particles. However, those inside the neuron probably participate in these processes only indirectly since they do not participate in the circulating currents. 


\section{Translocation of electrons in the brain}

The moment when the electrons gain enough energy to allow them to leave their position in the potential well, they, being in a quantum state, spill out of their original potential well in all directions, obeying the Schroedinger equation. The laws of classical physics must be replaced by the laws of quantum mechanics. It should be emphasized that there is no real physical motion of either a quantum particle or a matter wave involved in this process. In this situation, we say that the particles are in the quantum state. Each neuron is, therefore, a source of particles that, with their wave function and quantum state, may fill even remote areas of space. During this process, the particles may spread over the whole universe (which is mathematically possible), or at least throughout the brain. Schroedinger's equation determines the probability of finding that particle in different places. While in a quantum state, the particle has no defined position. The square of the absolute value of the wave function determines the probability of the particle being localized in a particular region of space.

Therefore, this hypothesis differs substantially from the electromagnetic theory of consciousness which is based on movements of classical electrons and electrical currents through the brain.

This process of translocation is somewhat complicated in the brain tissue where it takes place in the extracellular spaces which are narrow and extend to a considerable distance, up to the surface of the brain. Since these extracellular spaces in the brain are rather narrow, the arrangement resembles the theoretical so-called "particle in a box" problem.

The extracellular spaces are enclosed in firm cellular membranes. The volume of extracellular space is about $20 \%$ of the brain volume (Bruehlmeier et al 2003). It is connected with brain ventricles and with the subdural space and filled with fluid. All of the extracellular spaces throughout the brain are interconnected and allow the translocation of quantum waves. In this aspect, the brain resembles other physical systems in nature. The main idea of this section is based on the paper by Holub and Smrz (2002). These authors found a somewhat similar situation, from the viewpoint of quantum physics, in a completely different physical system. They studied and mathematically proved the penetration of submicroscopic particles through narrow gaps in rocks so that they can be detected in remote areas. According to Krcmar and Vylita (2002) the unusual mobility of solid particles in the Earth crust may be explained by several hypotheses, the one closest to the behavior observed in nature is based on quantum mechanics, on the translocation of particles along narrow spaces in the rocks.

Quantum mechanics requires that the wave function in the "narrow box" describing the probability of the position of a particle be modified in such a way that it reaches a zero value on the surface of the firm impenetrable walls of the extracellular space. The wave function has to be modified by a sharp decrease near the walls of the extracellular space. Solving Schroedinger's equation, the modification of the wave function across the intercellular space described above results in an exponential increase of the wave function along the narrow extracellular space. The narrow space may even be curved (Holub and Smrz, 2002).

There may be several possibilities to explain the role of particles in a quantum state in the brain activities. The possibility proposed here is based on the Schroedinger equation where restriction of the wave function in one or two dimensions leads to an extension in the remaining dimension(s). This restriction leads to the probability of particle localization increasing along the intercellular spaces. However, this localization has a statistical character.

\section{The decoherence of electrons in the brain}

The particles in the quantum state cannot be observed until they are collapsed. Particles in a quantum state may return to the classical physical form by the process of decoherence. Decoherence may be nearly instantaneous. In quantum theory, decoherence means a process of transition from the quantum to the classical physical state. An important aspect in this hypothesis is that we have to distinguish a quantum particle before decoherence and a classical electron after decoherence. The wave function may exist anywhere in the space and decoherence of the quantum waves may also occur anywhere in it. When the wave function decoheres, the particle is localized and bound to the brain structures.

This process of decoherence is still subject to a number of theories and we shall not be concerned with the details. Decoherence in the brain is too fast to sustain long-lasting coherent states. On the other hand, we expect that decoherence must be quite fast to achieve fast changes in the state of the brain. According to Tegmark (2000), decoherence in the relatively warm and wet environment of the brain appears within $10^{-13}$ to $10^{-20}$ of a second. This is necessary for the simultaneity of numerous interactions used to accomplish the binding of functions of various parts of the CNS. A quantum state particle may decohere in any position in space and change into a real physical particle.

Decoherence further depends on contact with the classical 
structure of matter in the environment. A crucial role in this process is therefore played by the natural environment. To prevent decoherence, there must be space available which is not occupied by any classical form of matter. Therefore, translocation requires empty space because decoherence is caused by the interaction of the quantum with the classical environment (Zurek, 2003).

If, therefore, we consider the intercellular spaces in the brain a "narrow box" in the sense of the Schroedinger equation, then another necessary condition is empty space. This is present between water and solute molecules in the cerebrospinal fluid of the extracellular space throughout the brain (Trincher, 1981). There are molecules of water and other particles in the extracellular space of the brain that may induce decoherence but the particles do not have to decohere if an empty space is available. Decoherence therefore has a statistical character. Also, where the intercellular gap opens into a wider space, classical physical particles are formed by the process of decoherence and are bound to the adjacent brain structure. The probability of particle localization is maximal in the vicinity of the point where the narrow gap widens. This is again explained by the Schroedinger equation. This point may be located in the vicinity of the synapses or at the end of the intercellular gap, at its opening to the intercellular space on the surface of the brain.

In these considerations, we cannot avoid the possibility of other quantum mechanical interpretations, such as electron tunneling through the areas where the energy is higher than the energy of the electron. However, we believe that such an alternative proposal would make the whole process even more difficult to explain.

\section{Functions of the translocated electrons}

When the wave function collapses, the particle is localized and bound to the brain structures. After decoherence, the electrons may induce a number of physiological events. Since the warm and wet environment is not homogeneous, the decoherence of electrons takes place differently in various cellular systems and brain areas. The mechanism proposed here, including limitations caused by insulating neuronal and other membranes and myelin, may participate in the decoherence of the particles in some preferred position. Still, this mechanism has a rather general character. We postulate that the electrons, after their decoherence, may cause several events:

1. Anywhere on the surface of the neuronal body or dendrite, they may directly boost the amplitude of the excitatory postsynaptic potentials and decrease the amplitude of the inhibitory postsynaptic potentials.
2. They may open the electrically sensitive ionic channels and increase the amplitude of the postsynaptic excitatory and inhibitory potentials.

3. In the triggering zones in the axon hillock, they may contribute to the generation of nerve impulses.

4. They may change some biochemical events within the cells and/or the cell membranes, e.g., by changing the configuration of the intramolecular double bonds. It may be isomerization of a relatively simple organic molecule, or some effect on the second messenger system.

5. They may affect the movement of synaptic vesicles to the surface membrane of the nerve endings which is induced by calcium ions, or something else that alters the excitation state of the target neuron. These and other possibilities may be elucidated experimentally by existing methods.

6. They may decohere anywhere in the brain tissue, thus form a complex field which will be described later.

7. We cannot exclude the possibility that they may decohereoutside the brain.

\section{The Interactions of Neurons in the Brain}

The human brain is composed of neurons and glia cells. The estimates of the number of neurons in the brain differ, and they reach values of up to 100 billion. Each neuron may be directly affected by as many as 10,000 other neurons at one time, usually through synapses. The number of synapses is enormous, estimated at 300,000 billion in the cerebral cortex. In the cerebral cortex, at least one quadrillion nerve cell transactions are executed each second (Crick, 1994). Still, all these components of the brain tissue work together and form a unified system. How is it possible?

The function of the central nervous system depends on many serial and parallel interactions of masses of individual neurons. In the brain, we may observe convergence and divergence, feedbacks, reverberations and circulating nerve impulses. The reverberating neuronal circuits may be rather long, lasting up to one second (Reinis, 1997) or more. All these events participate in the functioning of neuronal networks which contain millions of neurons and billions of synapses. Such sequences of neuronal firing are required to respond to the environment in a real, sufficiently short time. These data show the enormity of the number of neuronal interactions which must be handled by the brain.

The functions mentioned in this section may induce an increase or decrease in the firing of individual remote neurons. Therefore, the elementary functions of single neurons may be influenced by remote sources of electrons. 
Thus, we postulate that beside the classical interaction of neurons in the brain, there is a very general communication system involving the quantum state of electrons. It may participate in interactions of remote structures of the brain, in particular in the brain cortex, which contains about $75 \%$ of all neurons in the CNS.

There are several functions in the brain where such a general system may play a role in activities attributed to consciousness. In the first place, such a system may participate in the generation of global fields or transient synchronous assemblies which can be involved in the coordination of masses of neurons. That assures the unity of phenomenal consciousness. Therefore, such a system carries a more global informational access. Thus, for instance, the information carried in conscious or nonconscious mental states may be widely available. That guarantees a more unified and more integrated representation of reality. The real external objects and actions are usually presented to us in a multi-modal fashion that involves the integration of information from various sensory channels. The conscious experience shows us not isolated properties or features but objects and events. Also, a flexible and more sophisticated control may be provided. Conscious mental processes provide highly flexible and adaptive forms of control in novel situations and the unity of action. This system is presumably very fast, and therefore flexible, and forms the "stream of consciousness".

\section{EEG - window on the mind}

Supporting this hypothesis on the role of free electrons in the brain is our explanation of the generation of the electroencephalogram. The electroencephalogram reflects a general state of brain function, from the deepest coma to the highest levels of a conscious intellectual activity. It is, as stated by Nunez and Srinivasan (2006), a window on the mind. The literature concerning this subject is overwhelming. Correlations exist between EEG and cognitive mental actions and calculations, listening to music, speech, watching pictures, mental interpretation, reading, motor processes and other special brain activities. In early papers, the action potentials of individual neurons were thought to form the EEG (Adrian and Matthews, 1934). But, the action potentials of neurons hardly resemble the waves of the electroencephalogram. Their shapes are completely different, and the amplitude of the neuronal potentials is expressed in millivolts whereas the amplitude of the electroencephalogram waves is measured in microvolts. Most authors, including many textbooks, claim that the electroencephalogram is produced in the most superficial layers of the cerebral cortex by the flow of synaptic currents through extracellular space. The potentials are added by superposition. The synaptic currents form the "synaptic action fields".

Common theories of the origin of the electroencephalogram therefore assume that the waves of the electroencephalogram are generated by a millisecond scale modulation of synaptic transmission in the masses of neurons over a large area of the cortex (Eccles, 1984, Freeman, 2005, Nunez and Srinivasar, 2006). Eccles proposed that EEG activities are generated by summed postsynaptic potentials arising from the synchronized excitation of cortical neurons. Intracellular recordings from cortical neurons later demonstrated a close correspondence between EEG/LFP activity and synaptic potentials (Creutzfeldt et al., 1966).

The current view is that EEG waves are generated by synchronized synaptic currents arising on cortical neurons, possibly through the formation of dipoles (Nunez, 1981). The incoming excitatory signal at the synapse gives rise to a postsynaptic potential resulting from positively charged ions rushing into the cell. This leaves a relatively negative charge in the extracellular space in the vicinity of the synapse. The inward current at the synapse flows down the dendrite and ultimately moves outward across the cell membrane to sites distant from the synapse. The outward flow of positive charge leaves a relatively positive charge in the extracellular space. At this instant there is a dipole outside the dendrite, with a relatively negative charge at the distal part of the dendrite and a positive charge closer to the cell body. The summation of the dipoles formed at each of thousands of neurons creates an electrical potential detectable on the scalp.

In all these considerations, one factor was incompletely known, and that is a common controlling principle. Large scale cortical potentials are believed to arise from functional integration of synaptic current sources. These microsources related to individual synaptic actions are components of potentials described as mesosources forming intermediate scale dipole moments. The potentials everywhere in the vicinity of the cortex are expressed as the sums of contributions from all mesosources.

\section{Association of electron translocation with cortical synaptic activity}

Masses of electrons translocated by the mechanism described above and reaching the surface of the brain obviously influence the local micro- and mesosources generated by masses of local neurons. After translocation, a remaining small percentage of electrons decohere where the intercellular gap widens into the subdural space on the 
surface of the brain. The area where the intercellular gaps get wider is, in correlation with the Schroedinger equation, the area where an increased number of electrons decohere. Synaptic activity is modulated by these translocated electrons. Excitatory postsynaptic potentials become more negative, and inhibitory postsynaptic potentials become less positive. That affects the excitability of the neurons up to the point that they reach the firing level.

The amplitude of the waves of the electroencephalogram indicates how much electrical activity is going on beneath the recording electrodes. The postsynaptic potentials are modified by translocated electrons and together form an electric synaptic field. This field interacts, affects and is affected by local synaptic actions and local dipoles. The continuum of the EEG across large areas of the hemispheres is accomplished by this mechanism as well. Long and short interactions dominate local and global dynamic processes in the cortex. This may facilitate interactions between remote cell assemblies, providing an important mechanism for the functional integration underlying conscious experience. It seems obvious that shorter waves of the EEG originate in shorter reverberating circuits or smaller neuronal assemblies. The EEG wave frequency indicates how large the deeper reverberating circuits are. From this wavelength we may estimate the size of the utilized neuronal assemblies. The size of the neuronal assembly therefore correlates conversely with the frequency of the waves of the EEG. Related to this problem is the work of Pizzi et al. (2004). They studied colonies of neurons derived from human neural stem cells. They had two colonies carefully shielded one from another. Laser stimulation of one culture produced nerve impulses in not only in the same culture, but also nerve impulses in the second culture. This result indicates existence of some kind of quantum interaction similar to that we postulate.

\section{The brainstem potentials}

In order to estimate the neural sources of scalp potentials and electric fields, one has to solve the associated forward problem. The forward problem maps sources of EEG potentials, their location, strength, and orientation. An example of this is the brainstem auditory evoked potentials generated by short clicks administered to the ear. The first wave, wave 1 , is generated by discharges of the distal part of the cochlear nerve formed by about 40,000 nerve fibers. Not all of them fire at once, as shown during the averaging of the repeated responses. The averaged curve of wave 1 resembles most of all, the normal distribution curve. Wave 1 appears during the first millisecond after stimulus. During the first fifteen milliseconds, a number of waves may be recorded from the surface of the cortex. The impulses passing through the anatomically defined auditory pathway in the brain stem and inducing the cortical evoked potential reach the cortex gradually and later. This indicates that the waves recorded from the surface of the cortex have an origin deep in the brain and do not depend on neuronal connections between the source and area of recording. We have to answer the following question: How are the brainstem auditory potentials transferred into the cortex without passing through the auditory pathway? There is only one explanation. The brainstem auditory evoked potentials prove that a mechanism we are describing really functions in the brain.

\section{Is it possible to solve the Hard Problem of consciousness?}

As stated by Stapp (2004, p. 250), the problem of consciousness cannot be solved without considering quantum mechanics. The question is how to use quantum mechanics and what kind of dynamics is suitable for this task.

There are at least three advantages to this quantum approach: first, the temporary connection of various systems might be sufficiently fast; second, the connections may be quickly initiated and terminated; and third, quantum interactions may also help to explain subjective consciousness.

John Searle wrote:

"Mind is the name of the process, not a thing. That process is entirely caused by physiological events in the brain. It is not something mysterious that stands outside of the natural world. It is just part of our ordinary biological life."

Everything in the universe is considered to be made up of atoms and their components, molecules, force fields etc., but it seemed that the components of the mind and consciousness do not belong to any of these categories. It is believed that consciousness is real, but utterly unexplainable in any terms hitherto familiar to science. Thus, it stands as an extra fact that demands explanation. For a long time, researchers were talking about the explanatory gap and many believed that that is an absolute limit of our present understanding of the world and that we will never know.

The Hard Problem of Consciousness refers to the fact that the function of the brain, explained as a great information processor, may be eventually explored and analyzed to the slightest detail, but we are still unable to explain even the 
basic subjective sensations, those called qualia, such as perception of colors, sounds and shapes. How can a nonconscious substrate produce consciousness? Will we be able to bridge the gap? Everything described till now may explain several attributes of consciousness but the most essential problem, the subjective perception of reality, the Hard Problem of Consciousness.

However, the search for an answer is not entirely hopeless. In order to solve this problem, a question to ask is, which microparticle in the brain, or outside the brain, is able to communicate with other microparticles in the brain, and is able to transmit at least a small amount of information. Communication between particles is an elementary act important for the generation of a more complex phenomenon, mind and consciousness. A huge system of mind and consciousness may be constructed in the brain from submicroscopic communicating components.

The answer to this question is that practically all particles in the universe communicate with the others. There are many observations in quantum physics where some kind of communication between submicroscopic particles is demonstrated. Mutual exchange of information is common. It is, e.g., present in the thought experiment EPR (Einstein, Podolsky and Rosen, 1935) with its consequences. In this "spooky communication of two quantum particles at a distance", as it was called by Einstein, the particles were able to communicate and alter their state by a speed exceeding the speed of light. That connection has been demonstrated experimentally.

Similar verified principles, which are generally valid in nature, must function in the brain as well. Of several possibilities, it is first necessary to consider the electrons in the brain. There is an abundance of the free electrons (exoelectrons) in the brain, dependent on the function of neurons. They can be translocated anywhere, and they may be responsible for several functions described above.

The electrons that are released during the generation and spreading of the postsynaptic and action potentials and are then translocated throughout the brain, communicate their quantum state one to another. The interaction of electrons is described by the Pauli principle. The Pauli principle is an example of a general rule which applies not only to electrons but also to other particles with a halfinteger spin (fermions). No two neighbouring electrons at the same energetic level in an atom can have all quantum numbers identical. Pauli's exclusion principle indicates that two electrons may have three identical quantum numbers but the fourth quantum number must be different in order to become neighbours at one energetic level in the atom. Spin is a part of the quantum state of the electron, so two electrons with different spin have two different quantum numbers. However, the spin can take only two different eigenvalues.

The Pauli's exclusion principle is a part of one of the most basic observations of nature: neighbouring particles of half-integer spin must have antisymmetric wavefunctions. The Pauli exclusion principle was originally formulated for atoms, but it plays a role in a number of physical phenomena as well. It is responsible for the stability of matter. Molecules cannot be pushed arbitrarily close together, because the bound electrons in each molecule are forbidden from entering the same state as electrons in other molecules. Therefore, the objects do not collapse.

The Pauli principle obviously requires the existence of a mechanism by which an electron informs the other electrons about its own quantum number. This principle is manifest as an effective repulsion between any pair of identical electrons possessing the same set of quantum numbers. This is the basic fact of the Pauli principle. The electrons "feel" the presence of other electrons at a certain distance and respond to it.

This basic function of electrons is close to the human subjective feeling of "consciousness". We propose that this communication ability also represents the most basic unit of consciousness.

This information exchange is therefore, presumably, the most elementary basic unit of brain function. We may call it "protoconsciousness". However, this term has a number of meanings in literature. Here it is, simply, a communication mechanism of electrons, a powerful information function. That is transformed into an extremely complex structure of the human mind and eventually consciousness.

This is our proposal to how the Hard Problem of Consciousness may be solved. Methods similar to the construction of quantum computers may be utilized to give us the details of electron interactions depending on neuronal activity. The electrons have their characteristics of quantum interactions and at the same time they create the subjective feelings of consciousness. There is no gap. That is how we perceive the world.

\section{Discussion}

The explanation of consciousness lies in communication between electrons. Communication between electrons may be the most elementary building stone of subjective consciousness. At the same time that this communication between particles stands as a fundamental building mechanism of the universe, it aggregates into full-blown human consciousness under the conditions of the brain structure and function... 
The human mind is therefore a function of free electrons and their sensitivity and communication ability, in cooperation with the neuronal systems and assemblies. Subjective consciousness is a component of the mind and arises from the same electron sensitivity and other functions of the electrons, such as their translocation.

We have to stress that this model of the mind and consciousness is formed by two components. One is the mass activity of neurons responsible for the informational content of brain activity, and the second is the massive action of electrons. Together they produce the highest controlling system in the brain, the human mind, associated with consciousness. The human mind and consciousness therefore depend on the interactions of submicroscopic particles in the brain and, in particular, on the Pauli exchange principle.

Electrons in the brain are generated by neuronal activity and translocated throughout the brain. They form a field in the brain which is able to activate a number of neurons and get input from them. The motion of any one electron is strongly coupled to the motion of other electrons in the system. To describe the quantum mechanical behavior of electrons, it is necessary to calculate the many-electron wavefunction for the system.

This is a quantum effect acting bidirectionally and obviously in a rather complicated way. It must read all the components of the quantum numbers, refer them back to the electron which emitted the exchange force and both electrons must behave accordingly.

The activity of neurons generates a massive number of particles which is able to influence the actions of many target neurons. The affected target neurons generate another set of particles. The process goes forward indefinitely and very quickly and requires a fast decoherence rate. It renews itself continuously, whenever a new particle enters or leaves it. To put these considerations into an equation means to induce a differential equation for each electron in the brain. Our computers cannot do that.

Since the function of the brain changes with each fraction of a second, this high-level controlling system changes as well. Some considerations described previously indicate how numerous are the components of the whole system of the human mind and how quickly they alter their function. Inhibitory and excitatory mechanisms must cooperate in order to make this system meaningful.

A similar quantum mechanism probably plays a role in the binding problem of consciousness as well. According to Braitenberg and Schitz (1998), all neurons in the cerebral cortex are morphologically interconnected through two or three synapses Therefore, according to them, the cerebral cortex is a purely statistical ensemble of axons, dendrites and synapses. That obviously requires a higher controlling system organizing these ensembles. Braitenberg and Schitz themselves admit that their scheme which utilizes direct connections between all the neurons of the cortex, as some models of an "associative matrix" kind have, is functionally improbable. For this reason, we cannot expect that stimulation of one neuron will activate all the neurons in the cortex. But, on the other hand, the Pauli principle together with this anatomical arrangement means that the brain, or at least the cerebral cortex, forms a huge and powerful unified system able to produce the mind and, eventually, consciousness.

The brain does not function as a randomly assembled system. The diffuse and apparently haphazard arrangement of synaptic boutons along the branches of the axonal tree suggests a distribution of signals to large sets of other neurons. As a consequence of this, the condition for a cortical neuron to be activated must be the synchronous activity of many of its afferents. The brain, or at least the cerebral cortex, represents a powerful unit, where all neurons are interconnected morphologically, biochemically and by quantum connections, including the Pauli principle. All that assures the unity of function. We assume that this system contains not only excitatory relations, but also inhibitory ones. It must be limited in its activities by inhibitory influences, local hyperpolarizations Excessive firing must be prevented because it will interfere with the proper functioning of the brain.

Still, the explanation of consciousness and its content by our proposal seems to represent a certain paradox. Our human subjective "immaterial" thinking and reasoning is explained "by "material", i.e., physical mechanisms. Actually, there is probably no other possibility. Even if we admit the existence of an immaterial unifying principle, we have to understand that it may influence the "material" substrate of the brain only through some immaterial-to-material transition.

The hypothesis presented here may explain a number of phenomena. In the first place, the hypothesis indicates that the generation of mind and consciousness is based on a combination of quantum mechanisms, such as translocation of electrons and their interactions, with neuronal activity. The solution to the hard problem of consciousness also depends on elementary characteristics of the submicroscopic particles within the brain, in particular on the Pauli mechanism which may be a carrier of the subjectivity of consciousness. 
Therefore, we postulate that the quantum connection of many neurons plays a major role in the functioning of the brain. If every synapse were interpreted as a component in a strictly defined wiring diagram, it would be difficult to imagine how this system could work on its own. It has to be controlled somehow. We propose that this is a real purpose of the human mind, a homunculus, self, ego or superego, as this mechanism is called by various terminologies.

Therefore, any portion of the cortex may be informed about activity in the rest of the cortex, not only through direct fibre connections or by way of a few synapses, but also by our proposed quantum mechanism. A problem to be solved is, how some of these mechanisms enter subjective consciousness and some of them do not. The difference between mind and consciousness changes and the transition is not very sharp (Merikle, 2000). Some brain activities do not enter consciousness. It is possible that unconscious actions just involve a smaller number of neurons. The fields where large masses of neurons are activated simultaneously contain a conscious activity of the moment. In the next fraction of a second, this area containing consciousness moves somewhere else. Although there are several types of consciousness described in literature, this principle may perhaps be applied to all of them. These principles may represent a link bridging the subjective and objective components of the brain function. They may form a basis for a general reevaluation of the consciousness problem.

\section{REFERENCES}

Adrian ED (1941). Afferent discharges to the cerebral cortex from peripheral sense organs. J Physiol (London). 19: 159 - 191.

Adrian, ED and Matthews, BHC (1934). The interpretation of potential waves in the cortex JPhysiol (London). 80: 440-471.

Braitenberg V and Schitz A (1998). Anatomy of the Cortex: Statistics and Geometry. New York: Springer Verlag.

Bruehlmeier M, Roelcke U, Blauenstein P, Missimer J, Schubiger PA, Locher JT, Pellikka R, Ametamey SM (2003). Measurement of the extracellular space in brain tumors using $76 \mathrm{Br}$-bromide and PET favorite. J Nucl Med, 34: 1210-1218.

Chalmers DJ (1997). The Conscious Mind. In Search of FundamentalTheory, Oxford University Press, New York.

Crick F (1994). The astonishing hypothesis The scientific search for the soul. New York: Charles Scribner's Sons, Macmillan Publishing Company.

Creutzfeldt O, Houchin J (1974). Neuronal basis of EEG waves (In Handbook of Electroencephalography and Clinical Neurophysiology vol2 Part C, Ed In Chief A Remand, pp $5-55$,Amsterdam, Elsevier,
Eccles JC (1984). The cerebral cortex: theory of its operation (In: EC Jones and A Peterson Eds,Cerebral Cortex, Vol2 Functional Properties of Cortical Cells New York: Plenum.

Einstein A, Podolsky B, Rosen N (1935). Can quantum mechanical description of physical reality be considered complete? Physical Review 407: 777-780.

Freeman WJ III (2005). Origin, structure and role of background EEG activity 2 Analytic phase Clinical Neurophysiology 115: 2089 - 2107.

Gray CM and McCormick DA (1996). Chattering cells: Superficial pyramidal neurons contributing to the generation of synchronous oscillations in the visual cortex; Science, 276:101-113

Heisenberg W (1971). Physics and Beyond New York: Harper and Row.

Holub RF and Smrz PK (2002). Localization of a bound particle outside the potential well CanJPhys 250: 755-766

Huxley AL (1964). The Conduction of the Nervous Impulse (Thomas, Springfield, II, USA,

Krcmar B and Vylita T (2002). Unfilterable "geoaerosols", their use in the search for thermal, mineral and mineralized waters, and their possible influence on the origin of certain types of mineral waters' Environmental Geology $40678-682$.

Metzinger T (Ed (1985), Conscious Experience, (Exeter: Imprint Academic pp 3 - 37

Nunez PL (1981). Electric fields of the brain, Oxford University Press

Nunez PL and Srinivasan R (2006). Electric Fields of the Brain. The Neurophysics of the Brain Oxford University Press.

Pauli W (1980). General Principles of Quantum Mechanics (Berlin: Springer Verlag.

Penrose R (1994). Shadows of the mind. Oxford University Press .

Pizzi R, Fantasia A, Gelain F, Rossetti D, Vescovi A, (2004). Nonlocality in biological systems? An experimental approach CIFA News Jan-June, pp $16-18$.

Reinis S (1997). Some principles of decoding local neuronal systems Neural Network World, 7: 205-225.

Reinis S, Holub RH, Smrz P (2005), Quantum hypothesis of the brain activity and consciousness' (in Czech). Ceskoslovenska Fyziologie 54: $26-31$.

Stapp HR (2004). Mind, matter and quantum mechanics New York: Springer Verlag

Steriade, M and Amzica. F (1994). Dynamic coupling among neocortical neurons during evoked and spontaneous spike-wave seizure activity J Neurophysiol 72: 2051 - 2060.

Tegmark $M$ (2000). The importance of quantum decoherence in brain processes' Phys Rev E 61:4194-4206,

Trincher K (1981). The mathematic - thermodynamic analysis of water and the temperature range of life' Water Resources, 15433 448. 
S. Reinis

Zohar D (1990). The quantum self (London: Bloomsbury Publishing

Co.

Zurek WH (2003). Decoherence, einselection, and the quantum origins of the classical' Rev Mod Phys 75: 715-730. 